\title{
Aprendizado Acessível
}

Marcos D. do Nascimento ${ }^{1}$, Eder Furtado ${ }^{2}$, Bruno da S. Queiroz ${ }^{3}$, Lidiane C. e Silva $^{4}$, Francisco Carlos de Matos B. Oliveira ${ }^{5}$, David M. Ribeiro ${ }^{6}$, Cristiane M. de Souza Ferreira ${ }^{7}$, Michael G. de Almeida ${ }^{8}$

\author{
1, 2, 3, 4 Universidade Estadual do Ceará (UECE) \\ Caixa Postal 60741-000 - Fortaleza - CE - Brasil \\ ${ }^{5}$ Universidade de Fortaleza (UNIFOR) \\ Caixa Postal 60811-905 - Fortaleza - CE - Brasil \\ $6,7,8$ Universidade Federal do Ceará (UFC) \\ Caixa Postal 60020-180 - Fortaleza - CE - Brasil
}

\footnotetext{
devaner@dellead.com, eder_soares@dell.com, bruno@bsqinova.com, lidcastroddellead.com, fran.mb.oliveira@gmail.com, davidedellead.com, cristianeferreira@dellead.com, michael@dellead.com
}

\begin{abstract}
The Affordable Learning App, developed by the Distance Learning Lab for People with Disabilities (LE@D), presents itself as a solution for affordable distance education, available for tablets, smartphones and Android. The App has accessible learning objects with content translation for pounds and audio description of images. The entire layout and flow of navigation was planned and tested by a multidisciplinary team involving the deaf, blind and disabled. The App has accessibility features so that people with disabilities (PCD) can access resources that best meet their disabilities.
\end{abstract}

\section{Cenário de uso}

No Brasil, segundo dados do Censo 2010, realizado pelo IBGE - Instituto Brasileiro de Geografia e Estatística (Censo; 2010), existem cerca de 45 milhões de pessoas com algum tipo de deficiência (PCDs), o que representa $24 \%$ da população. Apesar da existência da lei de Cotas, lei 8213/91, diversas empresas continuam a sofrer multas por não conseguirem contratar pessoas com deficiência de acordo com o percentual exigido pela legislação, e alegam dificuldade no recrutamento de PCDs em função da falta de qualificação profissional. A falta de tecnologias, metodologias e materiais didáticos acessíveis para a devida qualificação desse público alvo é perceptível. Como consequência deste problema, a pessoa com deficiência enfrenta dificuldades para alocação no mercado de trabalho e é subjugada a trabalhos de baixa demanda intelectual.

O App Aprendizado Acessível, apresentado neste trabalho, é a plataforma móvel do Portal Dell Accessible Learning, um ambiente on-line de ensino a distância acessível para PCDs. Ambas as ferramentas foram desenvolvidas pelo Laboratório de Ensino a Distância para Pessoas com Deficiência - LE@D da empresa Dell Computadores, em parceria com a Universidade Estadual do Ceará - UECE e com o Instituto de Estudos, 
VI Congresso Brasileiro de Informática na Educação (CBIE 2017)

Anais dos Workshops do VI Congresso Brasileiro de Informática na Educação (WCBIE 2017)

Pesquisas e Projetos da UECE - IEPRO. O LE@D conta, atualmente, com cerca de 150 profissionais, entre eles doutores, mestres, especialistas, designers, programadores, testadores, pedagogos, intérpretes de Libras, audiodescritores, tutores, pesquisadores, dentre os quais 44 são pessoas com deficiência, que concebem e testam as tecnologias criadas.

Por meio do App são disponibilizados diversos cursos de capacitação profissional em Tecnologia da Informação, entre outras áreas, como Desenvolvimento de Software Java Básico, Desenvolvimento de Software Java Intermediário, Desenvolvimento de Software Java Avançado, Desenvolvimento de aplicativos Android, Administração de Banco de Dados Oracle, Gerenciamento de Projetos, Atendimento ao Cliente, entre outros, todos certificados pela Universidade Estadual do Ceará. Os cursos oferecidos por meio do App têm como objetivo facilitar o acesso à formação profissional, visando atender às especificidades de acessibilidade dos diversos perfis de PCDs no Brasil, e que, por outro lado, representam uma solução para que as empresas atendam à lei de cotas, atendendo uma necessidade do mercado e, consequentemente, incluindo PCDs no mercado de trabalho.

\section{Desenvolvimento}

No processo de concepção e criação do aplicativo, utilizou-se a técnica de design participativo (BARBOSA; SILVA, 2010), onde designers e um conjunto de usuários PCDs e não PCDs atuaram de forma colaborativa no desenho da interface e nos modelos de interação. O visual da interface foi elaborado usando as técnicas do Material Design, estilo de interface padrão do Android (CLIFTON,2015). Todos os ícones e componentes foram projetados ergonomicamente para que as pessoas com deficiência motora possam ter uma boa experiência de uso. O Material Design foi escolhido pela ampla disponibilidade de componentes e bibliotecas, assim como a fácil personalização, possibilitando maior produtividade ao processo de desenvolvimento.

\subsection{Tecnologias Utilizadas}

O aplicativo é executado em smartphones e tablets com o sistema operacional Android e foi desenvolvido em Java, que é uma das linguagens oficiais da plataforma, utilizando a IDE AndroidStudio. A versão mínima suportada é Android 4.1, API 16, chamada Jelly Bean (GOOGLE, 2017). Para executar o App, é necessário ter conexão com a Internet e um espaço disponível no dispositivo de no mínimo $12 \mathrm{MB}$. A aplicação possui funcionalidades relacionadas à visualização, download e upload de vídeos. Por exemplo, visualizar uma videoaula, ou gravar um vídeo com uma mensagem em Libras. Assim, é necessário que o dispositivo tenha suporte à gravação e à reprodução de vídeos no formato MP4.

Todo processo de comunicação e troca de serviços do App com a plataforma Web acontece via REST (do Inglês Representational State Transfer), como é mostrado na Figura 1. 


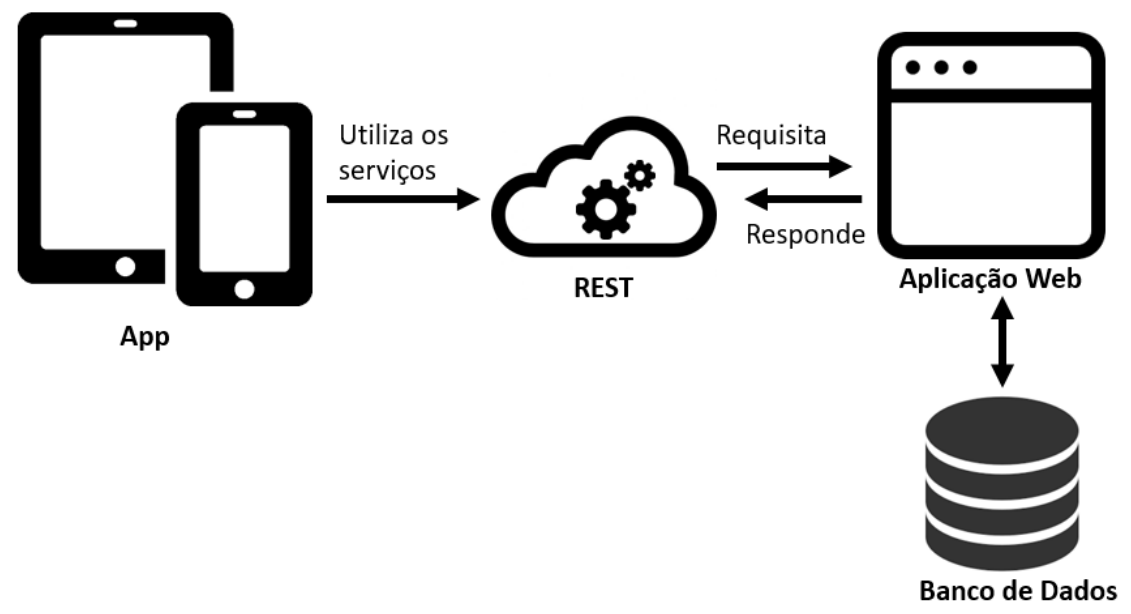

Figura 1 - Processo de comunicação e troca de serviços via REST.

O modelo de arquitetura REST utiliza o protocolo HTTP para troca de informações e serviços entre as aplicações (FERREIRA FILHO, 2011), desta forma, é possível a sincronização entre os status e estados das notificações, atividades e mensagens das aplicações Web e móvel.

\subsection{Testes e validação}

Testes funcionais: os testes funcionais são realizados por uma equipe de testadores, que são PCDs, com os perfis de deficiência física, visual, auditiva e baixa visão.

Testes de usabilidade: Além das funcionalidades testadas, também são realizados testes de usabilidade com o objetivo de identificar problemas de interface e o nível de satisfação do usuário com a interface e usabilidade do sistema. Para isso, são aplicados questionários de satisfação e testes de interação que permitem ao usuário ir catalogando os problemas à medida em que eles utilizam o App (BARBOSA; SILVA, 2010).

Para atender aos padrões internacionais de acessibilidade, durante a implementação do App atentou-se para todas as diretrizes da WCAG 2.0 (WCAG, 2008), além de utilizar o Scanner de acessibilidade da Google, que indica melhorias de acessibilidade para apps (GOOGLE, 2017).

\section{Apresentação do App}

O App Aprendizado Acessível é disponibilizado exclusivamente para os alunos. Com ele, os alunos poderão acessar os conteúdos das aulas, onde e quando quiserem, com maior flexibilidade e mobilidade.

Alunos PCDs contam com uma série de recursos de acessibilidade, como: alertas sonoros, tour adaptado para cegos, audiodescrição, tradução de conteúdos para Libras, ferramenta para solicitar tradução de correio e fórum, alto contraste, redimensionamento de fonte e comandos de voz. Essas funcionalidades permitem acessar os conteúdos de 
VI Congresso Brasileiro de Informática na Educação (CBIE 2017)

Anais dos Workshops do VI Congresso Brasileiro de Informática na Educação (WCBIE 2017)

forma mais dinâmica e acessível, eliminando possíveis barreiras de acesso à informação.

No ambiente do aluno, exemplificado na Figura 2, é mostrado um overview da situação do aluno em um determinado curso, mensagens de correio interno e calendário. Ao rolar para um curso, são carregadas as informações referentes ao curso em destaque. Para entrar em um curso, o aluno deve clicar sobre o curso em destaque. Nele, estão expostas a porcentagem de conclusão do curso, a situação de notas e frequência, novas mensagens do correio interno, próximos eventos e barra de acessibilidade com comando de voz, alto contraste e redimensionamento de fonte.

Ao selecionar um curso, é apresentada a lista de aulas. Conforme mostrado na Figura 2, todas as aulas pertencentes ao curso poderão ter os seguintes status:

- A iniciar: quando a aula ainda não está liberada ou não foi visitada pelo aluno.

- Cursando: quando a aula foi visitada pelo aluno, no prazo estabelecido pelo administrador do sistema, e as atividades que computam frequência ainda não foram realizadas.

- Finalizado: quando o aluno realizou todos as atividades que computam frequência.

- Não realizado: quando o aluno não realiza no prazo as atividades que computam frequência.

Ao selecionar uma aula, o aluno é direcionado para a aula selecionada, como mostrado na Figura 2, onde estão dispostos os botões de acesso para os objetos de aprendizagem que compõem a aula, sendo eles: videoaula, exercício, fórum, avaliação e podcast (os objetos de aprendizagem são disponibilizados mediante à configuração e agregação deles ao curso). Os ícones dos exercícios, fóruns e avaliações são alterados conforme o status de realização (A realizar, Realizado e Não realizado).

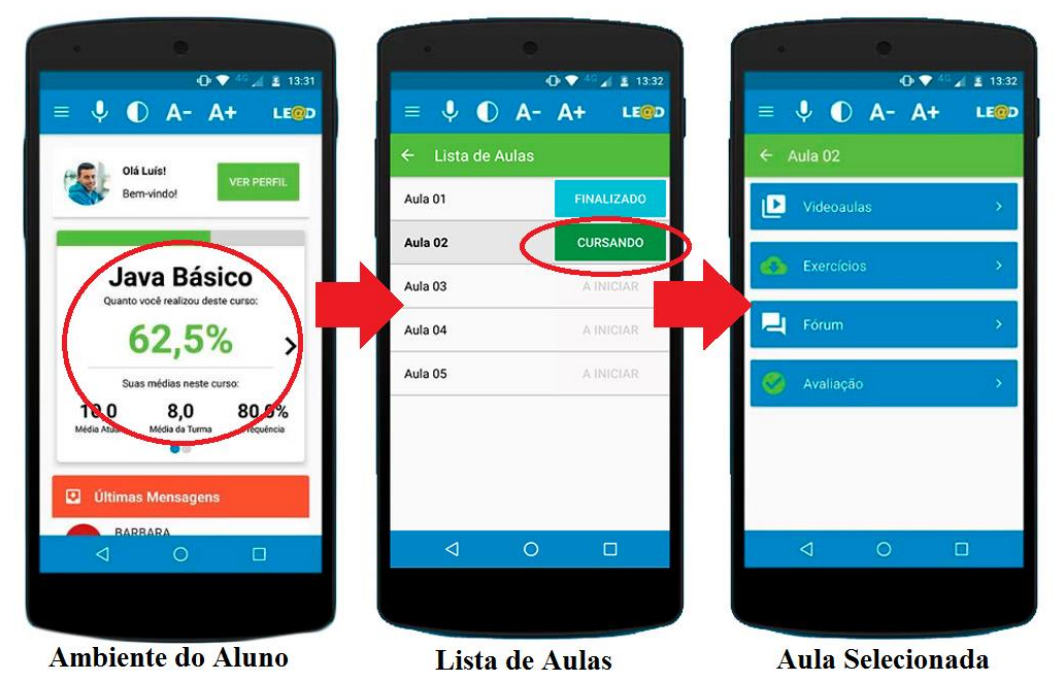

Figura 2 - Processo de comunicação e troca de serviços via REST.

Um vídeo com demonstrações do fluxo de navegação, dos objetos de aprendizagem e dos recursos de acessibilidade está disponível em: https://youtu.be/P-nWXftTTeE. 
VI Congresso Brasileiro de Informática na Educação (CBIE 2017)

Anais dos Workshops do VI Congresso Brasileiro de Informática na Educação (WCBIE 2017)

Existem algumas restrições em relação ao acesso e disponibilidade dos objetos de aprendizagem. São elas:

- O acesso aos conteúdos e ferramentas é restrito para alunos matriculados em um curso.

- A disponibilização dos cursos se dá por meio de parcerias com instituições privadas, governamentais e do terceiro setor.

- Para smartphones, o conteúdo das aulas está disponível apenas em forma de videoaula.

- Os alunos matriculados poderão fazer download do App por meio da Playstore, acessando o link: https://play.google.com/store/apps/details?id=br.com.lead.app.

\section{Considerações finais}

O aplicativo está em constante evolução. Todos os feedbacks dos usuários sobre problemas de uso e sugestões de melhorias do App são catalogados por uma equipe de suporte que direciona para os devidos responsáveis. Atualmente, trabalha-se para que o App atenda cada vez mais uma gama maior de pessoas com deficiência, realizando, para tanto, pesquisas para levantamento de requisitos específicos para cada deficiência ainda não atendida. Além da manutenção e evolução da versão para Android, também está sendo desenvolvida uma versão híbrida para que usuários de outros sistemas operacionais tenham acesso ao App.

Todos os esforços empregados visam atender e motivar pessoas com e sem deficiência, oferecendo-lhes um ambiente de aprendizado interativo, simples, intuitivo e acessível. Através de ferramentas como esta, a pessoa com deficiência pode ter acesso a novas oportunidades de aprendizado, abrindo-lhe novas possibilidades futuras. 
VI Congresso Brasileiro de Informática na Educação (CBIE 2017)

Anais dos Workshops do VI Congresso Brasileiro de Informática na Educação (WCBIE 2017)

\section{Referências}

CENSO, I. B. G. E. Instituto Brasileiro de Geografia e Estatística. Fornecido em meio eletrônico: [www. ibge. gov. br/home/estatistica/populacao/censo2010/]. Acessado em: v. 20, n. 03, p. 2012, 2010.

BARBOSA, Simone; SILVA, Bruno. Interação humano-computador. Elsevier Brasil, 2010.

CLIFTON, Ian G. Android User Interface Design: Implementing Material Design for Developers. Addison-Wesley Professional, 2015.

GOOGLE. Jelly Bean. Disponível em:

$<$ https://developer.android.com/about/versions/jelly-bean.html\#android-41> Acessado em: 05 de setembro de 2017.

FREITAS, Otávio. Serviços semânticos: uma abordagem RESTful. 2011. Tese de Doutorado. Universidade de São Paulo.

CALDWELL, Ben et al. Web content accessibility guidelines (WCAG) 2.0. WWW Consortium (W3C), 2008.GOOGLE. Ajuda do Android Accessibility Disponível em: < https://support.google.com/accessibility/android\#topic=6007234> Acessado em $12 \mathrm{de}$ setembro de 2017. 